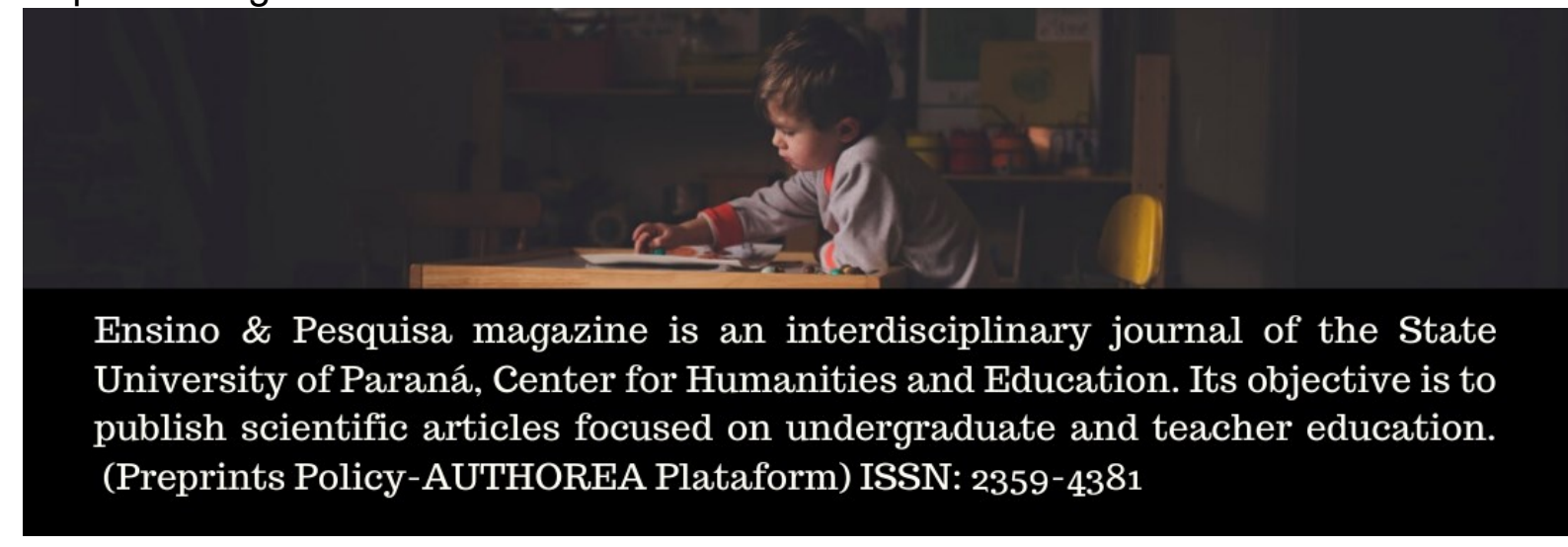

\title{
Representações sociais de saúde e doença entre acadêmicos de medicina
}

Rossana Andressa Mazzaro de Figueirêdo, Mestre em Mestre em Ensino nas Ciências da Saúde pela Faculdades Pequeno Príncipe, Docente do curso de Medicina da Universidade Positivo, rossana andressa@yahoo.com.br

Romilda Teodora Ens, Doutora em Educação, Docente do Programa de Mestrado e Doutorado em Educação da Pontifícia Universidade Católica do Paraná (PUCPR), Pesquisadora Associada da Fundação Carlos Chagas, participando do Centro Internacional de Estudos em Representações Sociais, Subjetividade - Educação (CIERS-ed), romilda.ens@gmail.com

Jaqueline Salanek Oliveira Nagel, Doutoranda em Educação pela PUCPR, Pedagoga da Rede Municipal de Ensino de Curitiba/PR, jaquenagel@gmail.com

Suely Grosseman, Doutora em Engenharia de Produção, Docente do Departamento de Pediatria da Universidade Federal de Santa Catarina - UFSC (Florianópolis - SC) e do Mestrado em Ensino nas Ciências da Saúde das Faculdades Pequeno Príncipe - FPP, sgrosseman@gmail.com

Leide da Conceição Sanches, Doutora em Sociologia, Docente do Programa de Mestrado em Ensino nas Ciências da Saúde das Faculdades Pequeno Príncipe- FPP, Docente das graduações em saúde da FPP, Membro do Comitê de Ética em Pesquisa da FPP, Membro do Grupo de Pesquisa em Sociologia da Saúde (UFPR), leide.sanches@,fpp.edu.br

Resumo: As representações sociais de Saúde e Doença e das causas para o adoecimento são diversas e recebem a influência do contexto sócio-histórico-cultural. O objetivo deste estudo foi conhecer as representações sociais de saúde e doença partilhadas por acadêmicos do curso de medicina. Abordagem metodológica foi qualitativa, com referencial teórico das Representações Sociais e dos pressupostos da Teoria do Núcleo Central (ABRIC) e a análise foi a de conteúdo. Participaram desse estudo o total de 336 acadêmicos: 50 ingressantes, 176 do sexto semestre e 110 do décimo segundo semestre do curso. Os resultados evidenciam que o significado da palavra saúde para os participantes de pesquisa está associado a um conceito mais amplo de bem-viver, com evocações tais como: bem-estar, felicidade, alegria, equilíbrio, paz, tranquilidade, satisfação, energia, disposição e plenitude. As palavras classificadas dentro da área temática do modelo ampliado de saúde foram: qualidade de vida, família, social, biopsicossocial, espiritual, promoção e prevenção. De forma antagônica, os significados da palavra doença foram relacionados à limitações do viver, com evocações como mal-estar, tristeza, sofrimento, dor, ruim, depressão, dificuldade, desconforto, cansaço, medo, ansiedade, solidão, fragilidade, angústia e incapacidade. A formação médica demonstrou propiciar a esses estudantes um contato conceitual com um modelo ampliado de saúde, entretanto, observou-se que, a aproximação é teórica e tênue. A relevância de alguns temas importantes para 
a formação médica ocupa espaço marginal nas representações sociais de saúde e doença dos estudantes desta pesquisa.

Palavras-chave: Processo Saúde-Doença; Saúde; Doença; Estudantes de Medicina.

\section{Social representations of health and illness among medical students}

Abstract: As social representations of Health and Action and causes for illness are diverse and are influenced by the socio-historical-cultural context. The aim of this study was to know how social representations of health and diseases shared by medical students. Methodological approach was qualitative, with theoretical reference of Social Representations and assumptions of Central Core Theory (ABRIC) and an analysis was performed with content. A total of 336 students participated in this study: 50 new entrants, 176 semesters and 110 second semester of the course. The results show the meaning of the word health for the research participants is associated with a broader concept of well-being, with evocations such as well-being, happiness, joy, balance, peace, tranquility, satisfaction, energy, disposition and fullness. The words classified in the area of thematic health model were: quality of life, family, social, biopsychosocial, spiritual, promotion and prevention. In an antagonistic way, the meanings of the word disease were related to limitations of living, with evocations such as uneasiness, sadness, suffering, pain, bad, depression, difficulty, discomfort, tiredness, fear, anxiety, loneliness, fragility, anguish and disability. Medical education has been shown to provide these students with conceptual contact with a broader model of health; however, an approach is theoretical and tenuous. The relevance of some important topics for medical education occupies a marginal space in the social representations of health and disease of the students of this research.

Keywords: Health-Disease Process; Health; Disease; Medicine student.

Submissão: 2019-12-21. Aprovação: 2020-03-11. Publicação: 2020-04-17

\section{Introdução}

As representações sociais de Saúde e Doença e das causas para o adoecimento são diversas e recebem a influência do histórico, das crenças, valores, expectativas pessoais e postura diante da vida, ou seja, do contexto sócio-histórico-cultural (CZERESNIA; MACIEL; OVIEDO, 2013).

Segundo a teoria das Representações Sociais a realidade é representada pelo indivíduo e reconstruída a partir dos sistemas cognitivos, da perspectiva simbólica e dos valores. Deste modo, entende-se que os fenômenos são compreendidos e comunicados de modo particular a cada indivíduo e que isso influenciará na preparação para uma ação, que não apenas guia os comportamentos, mas, sobretudo, remodela e reconstitui para si o arranjo dos elementos do ambiente no qual o comportamento ocorre (MOSCOVICI, 2015).

Ao longo dos dois últimos séculos, houve grande desenvolvimento técnicocientífico e valorização do tecnicismo. A visão do processo saúde-doença com abordagem biomédica predominou, com fragmentação do conhecimento e uma tendência à superespecialização na formação e prática médicas. O aparato tecnológico para diagnóstico e tratamento de doenças ganharam espaço crescente na estruturação dos serviços médicos e interpuseram-se entre o médico e o paciente. Em meio à complexidade do sistema, foi

Ensino \& Pesquisa, União da Vitória, v. 18, $n^{\circ}$ 1, p. 121-135, jan./abr., 2020. 
pouco considerada a subjetividade da pessoa que adoecia, o que limitou a qualidade do cuidado prestado. A formação médica era dividida em básica e profissionalizante, com abordagem pedagógica predominantemente teórica nos 4 a 5 primeiros anos do curso e o principal cenário de aprendizagem de prática era o hospital.

Nas últimas décadas, a partir da Constituição de 1988, o governo brasileiro garantiu acesso à saúde a todos os cidadãos brasileiros, por meio da criação do Sistema Único de Saúde (SUS). Para que os profissionais médicos se tornassem competentes a atuar em todos os níveis de atenção à saúde, era preciso alinhar a formação médica às necessidades de saúde da população e do SUS. Foram lançadas as Diretrizes Curriculares para os cursos de graduação em Medicina (DCNCGM) em 2001, para que o futuro profissional médico, tivesse uma formação generalista, com perfil humanista, crítico, reflexivo e ético, com competência para atuar em todos os níveis de atenção à saúde, baseado em um Conceito Ampliado de Saúde, e com responsabilidade social (BRASIL, 2014). As DCNCGM estabeleceram a necessidade de integração entre ciclo básico e clínico, uma formação generalista, aprendizagem em cenários de prática diversos, uma abordagem pedagógica significativa e maior tempo de prática no internato.

Em 2014, estas diretrizes foram modificadas para alinhamento da formação ao Programa Mais Médicos. De acordo com o descrito no Capítulo III, Artigo 23 das DCN para o Curso de Graduação em Medicina, os conteúdos fundamentais a serem abordados na educação médica devem se pautar no modelo ampliado de saúde, ao abarcar a compreensão dos determinantes sociais, culturais, comportamentais, psicológicos, ecológicos, éticos e legais, nos níveis individual e coletivo, do processo saúde-doença.

Apesar da influência das diretrizes na transformação da visão do processo saúdedoença na formação médica, essa visão é permeada pelo pensamento sociocultural no qual o acadêmico, profissionais do ensino e dos serviços de saúde e a própria comunidade se inserem.

\section{Método}

Estudo de abordagem qualitativa e fundamentado nas Representações Sociais, sob o aporte da Teoria do Núcleo Central, proposta e desenvolvida por Abric (2000). Os participantes foram acadêmicos de medicina ingressantes, os quais ainda não haviam iniciado as atividades acadêmicas do primeiro semestre (e por isso denominado como 
semestre 0), do $6^{\circ}$ semestre e do $12^{\circ}$ semestre de quatro Instituições de Ensino Superior (IES) da cidade de Curitiba/PR no ano de 2018. Estes semestres foram escolhidos, por possibilitarem caracterizar o conceito do processo saúde-doença ao ingressar na escola médica, no meio do processo de formação e ao seu final. O total de 336 acadêmicos aceitaram participar da pesquisa: 50 ingressantes, 176 do sexto semestre e 110 do décimo segundo semestre do curso.

A coleta das informações foi feita com questionário autoaplicado contendo variáveis sociodemográficas e semestre de matrícula. Foi solicitado aos participantes a livre evocação de palavras através do Testes de Evocação Livre de Palavras - TELP, para analisar a relação ao termo "saúde" e "doença". Ao final investigou-se a representação da doença através da questão "Para você o que causa o adoecimento?".

A análise dos dados quantitativos foi feita através de estatística descritiva. A análise das informações nos TELP foi realizada iniciando com a lematização dos termos evocados como recomendam Wachelke e Wolter (2011), seguida pela análise prototípica e de similitude por meio do software IRAMUTEQ - Interface de $R$ pour les Analyses Multidimensionelles de Textes et de Questionnaires.

A análise prototípica, também chamada análise das quatro casas, provê a força e a organização das evocações, sendo uma das técnicas mais difundidas para o estudo de Representações Sociais. A partir dessa, é possível identificar a estrutura da representação por meio de parâmetros de frequência e ordem da evocação de palavras, o provável núcleo central e o sistema periférico da representação social (CAMARGO; JUSTO, 2013).

A análise de similitude é uma forma de classificação dos "termos de uma produção discursiva com base no coeficiente de semelhança" e verificação do grau de ligação entre esses termos. Nessa análise, entende-se que dois itens serão mais próximos na representação, quanto mais elevado o número de indivíduos que os tratem da mesma maneira; em seguida, calcula-se um coeficiente de contingência que é um índice de semelhança clássico (FLAMENL, 1986). A partir do grau de conectividade entre as palavras, o software IRAMUTEQ permite uma representação gráfica por meio de uma árvore, a qual, apesar de não possibilitar a observação de todas as conexões existentes entre as palavras evocadas, permite a das que possuem maior grau de conectividade.

A sistematização dos dados provenientes do corpus monotemático de respostas curtas sobre a causalidade do adoecimento foi realizada manualmente através da análise de

Ensino \& Pesquisa, União da Vitória, v. 18, nº 1, p. 121-135, jan./abr., 2020. 
conteúdo preconizada por Bardin (2016). Inicia-se esta análise com separação dos segmentos de texto em unidades de significação, que são organizados em categorias. Posteriormente, foram reagrupadas as categorias com a análise do TELP.

A pesquisa foi aprovada pelo Comitê de Ética em Pesquisa - CEP/FPP sob o parecer n. 2.310.043.

\section{Resultados e Discussão}

\section{Perfil dos participantes}

Dentre os acadêmicos, 135 eram do sexo masculino (40,2\%) e 201 do feminino $(59,8 \%)$. A faixa etária predominante foi entre 21 e 25 anos, na qual estavam alocados $n$ participantes $(60,1 \%)$, que aumentou progressivamente de acordo com o semestre em que estavam matriculados. Em relação à cor, 300 participantes declararam a cor de pele como branca (89,3\%), 22 amarela (6,5\%), 12 pardas (3,6\%) e 2 negra (0,6\%). A naturalidade da maioria, 236 participantes $(64,5 \%)$ eram de cidades do Sul do Brasil, entretanto, participaram da pesquisa acadêmicos provenientes de todos os estados e um deles de outro país. Dentre os acadêmicos 240 (71.4\%) possuem religião, sendo que 168 (50\%) se declararam católicos, 42 (12,5\%) evangélicos, 14 (4,2\%) espíritas e menos de (quantas pessoas?) $1 \%$ judeu, afro-brasileiro, mulçumano ou oriental. A medicina foi o primeiro curso superior cursado por $86 \%$ dos participantes.

\section{Representações Sociais de Saúde e Doença e de causas para o adoecimento}

Entre os participantes, os núcleos temáticos das representações sociais sobre saúde e doença foram: Valorização da Doença, Subjetividade do Adoecimento, Locais de Atenção à Saúde e o Hospitalocentrismo, Saúde e Doença como Medicina, Modelo Ampliado de Saúde, Estilo de Vida e Aspectos relacionados aos desfechos da doença. A seguir são apresentados cada um destes núcleos temáticos, apresentados como subitens em negrito itálico.

\section{Valorização da doença}

Ensino \& Pesquisa, União da Vitória, v. 18, $n^{\circ}$ 1, p. 121-135, jan./abr., 2020. 
No TELP tanto a palavra "saúde" foi evocada a partir do termo indutor "doença" (25 acadêmicos), quanto a palavra "doença" ou a expressão "ausência de doença" foi evocada a partir do termo indutor "saúde" (73 acadêmicos). A maior frequência na evocação da doença em detrimento da evocação de saúde pode estar relacionada ao maior enfoque biomédico durante a formação, com priorização do estudo da doença, do seu processo de diagnóstico e de cura, e menor ênfase na prevenção e promoção da saúde.

A evocação do binômio saúde-doença foi maior entre os participantes do sexto e décimo-segundo semestres. Isso pode estar relacionada ao fato de este ser o tema central de estudo advindo da formação acadêmica, e, naturalmente, estas palavras passarem a fazer parte das interações verbais em seu cotidiano.

Quanto à causalidade do adoecimento, alguns participantes associaram à falta de saúde (P299) ou à "doença” (P270), de forma similar ao que ocorreu nos testes de evocação.

Para outros, os significados tiveram foco biomédico $(6,5 \%)$, como por exemplo funcionamento inadequado do corpo humano (P27) entre os ingressantes, e transtorno orgânico imenso (P241), alteração estrutural, funcional ou fisiológica do organismo transitória ou permanente (P278), microrganismos (P91; P190; P194; P226), infecções (P103; P172), parasitas, bactérias, fungos (P105; P196), patógenos (P164; P184), agente infectante (P201), agentes etiológicos (P106) e erros inatos (P 106), entre os acadêmicos do sexto e no décimo-segundo semestres.

Esta maior especificidade de fisiologia, agentes infecciosos e genética apontam para os significados de adoecimento adquiridos ao longo da formação.

Alguns participantes associaram ainda ao adoecimento uma culpabilização, como por exemplo falta de vontade, à quem adoece. (P294).

\section{Subjetividade do adoecimento}

Os significados da palavra saúde para alguns participantes foram associados a um conceito mais amplo de bem-viver, com evocações tais como: bem-estar, felicidade, alegria, equilíbrio, paz, tranquilidade, satisfação, energia, disposição e plenitude. 
De forma antagônica, os significados da palavra doença foram relacionados à limitações do viver, com evocações como mal-estar, tristeza, sofrimento, dor, ruim, depressão, dificuldade, desconforto, cansaço, medo, ansiedade, solidão, fragilidade, angústia e incapacidade.

O termo com maior força de evocação da palavra "saúde" foi bem-estar ligado, que compôs o núcleo central de todos os quadros resultantes da análise prototípica gerada pelo termo indutor "saúde". Os termos com maior força de evocação da palavra "doença" tristeza e dor.

Quanto à causalidade do adoecimento, aspectos psicológicos foram mencionados entre participantes do semestre (40\%), do sexto semestre $(50 \%)$ e do do décimo-segundo semestre (53\%).

Os aspectos subjetivos foram enunciados tanto como multicausalidade do adoecimento, quanto como unicausalidade, como por exemplo: quando a pessoa se entrega a um problema de saúdel emocional e perde a esperançalforça (P2); estado emocional e mental debilitados (P6); como decorrência do pensamento negativo (P325), principalmente o fator psicológico (P317), o adoecimento é a somatização do nosso estado emocional que reflete no sistema imunológico no nosso corpo, na nossa organização orgânica. Todo o conjunto tem que estar bem para que não haja adoecimento” (P205).

\section{Locais de atenção à saúde e o hospitalocentrismo}

A partir dos termos indutores saúde e doença, foram evocados os seguintes locais e sistemas de atenção à saúde: Hospital, UBS (Unidade Básica de Saúde), UTI (Unidade de Terapia Intensiva) e UPA (Unidade de Pronto Atendimento), SUS (Sistema Único da

Saúde). A palavra Hospital foi a mais evocada entre os locais que prestam atendimentos entre os participantes de todos os semestres e a força de sua evocação foi menor no sexto período do que nos demais períodos analisados.

Dentre os participantes, três acadêmicos do sexto período evocaram a palavra UTI (Unidade de Terapia Intensiva) e um deles as Unidades de Pronto Atendimento (UPA). Salienta-se que nenhum acadêmico evocou pontos da rede de atenção à saúde relacionados à saúde mental como Centro de Atenção Psicossocial (CAPS), Hospital Psiquiátrico, ou os

Ensino \& Pesquisa, União da Vitória, v. 18, $n^{\circ}$ 1, p. 121-135, jan./abr., 2020. 
intersetoriais, como Centro de Referência de Assistência Social (CRAS), escolas, creches, entre outros equipamentos.

A UBS passa a ser parte da composição da RS, porém, em sua periferia e em duas das respostas na causalidade do adoecimento em acadêmicos do sexto período, a referência à Atenção Primária da Saúde (APS), a qual tem a UBS como seu principal local de atenção: falta de prevenção, informação e APS (P68); atendimento falho na APS, carência de campanhas de prevenção e promoção, sistema de saúde pouco eficiente, foco na doença e não no adoecer (P149).

A força de evocação da palavra Hospital pode ter sido menor no sexto semestre do que nos demais porque até essa etapa do curso o contato dos estudantes com o hospital é pequeno, e nos primeiros semestres eles começam a conhecer outros pontos da rede de atenção, como as Unidades Básicas de Saúde (UBS).

\section{Saúde e doença como medicina}

A medicina apareceu como um dos prováveis núcleos centrais das representações sociais de saúde e doença nos participantes da pesquisa. Por um lado, isto pode ser devido à medicina ser a profissão por eles escolhida, e por outro lado, pode ter relação com o paradigma biomédico vigente, o qual tem como uma de suas características o medicalocentrismo.

Nas respostas sobre a causalidade do adoecimento, duas acadêmicas do período 0 refletiram sobre a importância da integralidade do atendimento médico e do olhar humano do médico frente ao paciente. Em suas palavras:

O adoecimento, na minha opinião, não está necessariamente ligado somente a uma causa externa. Pode ser esse o caso, contido geralmente essa causa externa é a propulsora de um adoecimento interno, mental na maioria. Isso é comprovado na pratica por médicos que deixaram de analisar somente uma área do corpo, e passaram a tratar o paciente como um todo. Esse tipo de análise crítica ainda, infelizmente, não é empregada em sua plenitude no ocidente. Entretanto, aqueles que não o aplicam se deparam com uma frustração no processo de cura do paciente, pois sem um trabalho mental, também, as doenças são reincidentes. Todavia não necessariamente na mesma área corporal, assim o médico que não avalia o todo as trata independentemente sem efeito (P49). 


\section{Modelo ampliado de saúde}

As palavras classificadas dentro da área temática do modelo ampliado de saúde foram: qualidade de vida, família, social, biopsicossocial, espiritual, promoção $e$ prevenção. Pelas definições do modelo ampliado, caberiam dentro deste núcleo temático, os núcleos intitulados no presente estudo como, estilo de vida e aspectos subjetivos do adoecimento. Entretanto, pela força que as evocações tiveram sobre estes e por estarem entre as palavras dos prováveis núcleos centrais de cada RS, optou-se por alocá-los separadamente.

Para o termo indutor "saúde", as palavras social e biopsicossocial não foram evocadas pelos participantes do semestre 0 , e passam a compor a representação, em quadrantes periféricos entre os do sexto e décimo-segundo semestres. No comparativo entre os semestres de formação e a citação dos aspectos sociais relacionados à causalidade do adoecimento, nota-se que apenas $10 \%$ dos estudantes do semestre citaram esses aspectos, enquanto $35 \%$ do sexto período e do décimo-segundo período (53\%) os mencionaram.

Quanto ao conteúdo das respostas relativas aos aspectos sociais, a maioria dos acadêmicos as inserem no contexto de multicausalidade, seja por integrarem explicações do modelo biopsicossocial, ou ao citarem os fatores coadjuvantes sociais, como alimentação, emprego, saneamento básico e acesso aos serviços de saúde. Entre os acadêmicos do sexto semestre aparecem novas unidades de significação como: economia, politica, educacional, sistema de saúde, cultura e marginalização.

Infere-se que os acadêmicos participantes do estudo aprofundam a reflexão sobre os determinantes sociais durante a sua formação, uma vez que passam a ser nomeados e explicados em suas respostas.

A evocação das palavras social e biopsicossocial como razão para o adoecimento, remetem à possibilidade de ampliação do modelo paradigmático para a compreensão de saúde e doença.

A palavra família compõe a segunda periferia das Representações Sociais de Saúde e de Doença nos períodos 0 e 6 e a zona de contraste do período 12. Aspectos familiares relacionados à causalidade do adoecimento foram citados com frequência pelos acadêmicos do décimo-segundo semestre. No total, 14 acadêmicos a referenciaram, e 
destes, quatro $(20,5 \%)$ citaram aspectos biomédicos relacionados ao tema com a menção da hereditariedade para o adoecimento, com respostas que continham os termos "predisposições familiares" (P216), "heranças familiares" (P221) ou "história familiar" (P234 e P324,). Os demais acadêmicos referenciaram aspectos próprios do relacionamento e da dinâmica familiar, por meio de expressões como "contexto familiar" (P70; P77;P168; P315 ), "abandono familiar" (P88, 2018), "falta de apoio familiar" (P325), e também, além de menção às famílias, como sistemas interrelacionados ao seu contexto sócio cultural, como iulstrado a seguir: às condições de educação, cultura, finanças, as quais estão submetidas às famílias, o que influenciam seus laços e relacionamentos, seus hábitos e seu acesso a serviços de saúde (P231). A relação entre a pessoa que adoece e sua família foi citada como fator promotor ou protelador do adoecimento, como ilustrado a seguir:

o adoecimento está muito relacionado à maneira como a pessoa enferma, durante anos veio sendo tratada psicologicamente na família, além disso, como ela se sente na família. [...] Médicos devem tratar a alma do enfermo, que se encontra adoecida pelos problemas familiares, portanto, talvez um bom dia dado, pode mudar a vida de um paciente. (P47)

Em estudo desenvolvido sob a perspectiva fenomenológica para compreender os sentidos atribuídos ao processo saúde e doença entre adolescentes, usando a técnica de grupo focal, evidenciou a qualidade das relações intersubjetivas como essencial, em especial, as relações familiares conflituosas ou harmônicas como influenciadoras da saúde e da doença (BULGARELI et al., 2017). Além disso, Cromack e colaboradores (2006) também investigaram adolescentes e encontraram a palavra família na Zona de Contraste das evocações geradas pelo termo indutor "saúde".

A "Qualidade de vida" (QV), não foi evocada entre os acadêmicos do semestre 0 e integrou o provável núcleo central da RS a "saúde" entre os acadêmicos do sexto semestre. Nas questões sobre a causalidade do adoecimento, 16 acadêmicos abordaram aspectos de como o adoecimento interfere na qualidade de vida; de acordo com o acadêmico do décimo-segundo, a causalidade do adoecimento é "um processo de perda de algum parâmetro físico, mental, social, espiritual que leva a um prejuizo na qualidade de vida." (P277).

Ensino \& Pesquisa, União da Vitória, v. 18, $n^{\circ}$ 1, p. 121-135, jan./abr., 2020. 
A expressão "qualidade de vida ligada à saúde", tradução da expressão inglesa Healthrelated Quality of Life, tem sido utilizada para ser distinguida da QV, em seu significado mais geral. No contexto da área clínica o interesse tem sido, geralmente, naqueles aspectos da QV que são ou estão sendo influenciados pela ocorrência ou tratamento de doenças ou traumas (FAYERS, 2000).

Dentre os participantes, 5 acadêmicos mencionaram aspectos relacionados à espiritualidade, sendo ela incluída dentro de um conjunto de fatores, como por exemplo a causalidade do adoecimento é "multifatorial, desarranjo de tudo que nos torna humanos: psíquico, emocional, espiritual, biológico, social, cultural”. (P174)

No momento da coleta de informações, as IES nas quais os participantes estavam matriculados, não dispunham de disciplinas ou módulos específicos relacionados à temática da espiritualidade.

A dimensão espiritual da vida humana vem encontrando espaços crescentes nas comunidades acadêmicas. Braghetta (2017) estima que atualmente sejam publicados em média 7 artigos por dia sobre o tema. Essa introdução é fruto da progressiva quebra de vários paradigmas referentes ao papel do médico. Entretanto, a inclusão da espiritualidade nos currículos médicos nacionais é incipiente e praticada por uma minoria de IES. Em levantamento com 47,4\% das escolas médicas brasileiras, apenas 10,4\% dos cursos de graduação dispõe de disciplinas dedicadas ao ensino de espiritualidade e saúde, conforme Lucchetti e colaboradores (2012). O contato dos acadêmicos desde o primeiro ano do curso com estímulos que levem à compreensão da influência da dimensão espiritual na formação médica de forma humanista, reflexiva e crítica, otimiza a compreensão da integralidade do cuidado médico e promove atitudes empáticas e de compaixão (REGINATO; BENEDETTO; GALLIAN, 2016).

Ao termo indutor "saúde" e a palavra "promoção" aparecem no sistema periférico dessa RS entre os participantes do sexto período, com 9 evocações, e pela árvore máxima gerada pela análise de similitude observa-se a tendência em ser evocado com a palavra prevenção. O termo prevenção de doença apareceu como provável núcleo central da RS "saúde", entre os participantes do $6^{\circ}$ semestre. Nos demais períodos ele aparece como elemento do sistema periférico.

Ensino \& Pesquisa, União da Vitória, v. 18, nº 1, p. 121-135, jan./abr., 2020. 


\section{Estilo de vida}

De acordo com a Organização Mundial da Saúde (2004), Estilo de Vida é o conjunto de hábitos e costumes que são influenciados, modificados, encorajados ou inibidos pelo prolongado processo de socialização. Esses hábitos e costumes incluem indicações sobre as consequências do uso de substâncias tais como o álcool, fumo, chá ou café, hábitos de sono, dietéticos, bem como de práticas de exercício, por suas importantes implicações para a saúde e têm sido objeto frequente de investigações epidemiológicas. Os demais aspectos citados na definição acima da OMS não foram encontrados nas evocações geradas pelos termos indutores "saúde" e "doença".

\section{Intervenções terapêuticas sobre a saúde e a doença}

No núcleo temático Intervenções Terapêuticas, foram incluídos os agrupadores: remédio, cirurgia e reabilitação.

As evocações referentes ao agrupador remédio foram associadas pelos participantes mais frequentemente à palavra doença e a análise de similitude demonstrou que esse elemento esteve associado à palavra Hospital em todos os períodos do curso de Medicina. As palavras cirurgia e reabilitação situaram-se no sistema periférico da RS doença.

A falta ou negligência de tratamento foram referidas por 3 acadêmicos como causadoras de adoecimento, como segue: "ausência de prevenção, negligência a tratamentos, falta de autocuidado" (P121, 2018); "Falta de cuidado e tratamento adequado" (P8, 2018); "Falta de disseminação de informações, imunidade baixa do paciente, falta de infraestrutura das cidades e dos hospitais, falha na capacitação dos médicos, falha em diagnósticos, rotina estressante, remédios ineficazes...” (P28, 2018)

$\mathrm{O}$ achado da vinculação entre doença e remédio é comum na literatura (OLIVEIRA, 2001; GROSSI, 2012). Essa relação foi constatada na análise prototípica e na análise de similitude: hospital e remédio, que apontam características importantes do modelo de saúde reducionista biomédico: hospitalocentrismo e medicalização.

\section{Considerações finais}

Ensino \& Pesquisa, União da Vitória, v. 18, $n^{\circ}$ 1, p. 121-135, jan./abr., 2020. 
Investigar as representações sociais de saúde e doença de acadêmicos de medicina substanciou a reflexão, tanto sobre os modelos paradigmáticos que norteiam essas concepções, quanto embasou ponderações acerca da formação médica e da sua adequabilidade às DCNCGMs (BRASIL, 2014).

Alguns temas relevantes para a formação médica foram, embora em menor escala, evocados ou mencionados pelos acadêmicos nas respostas sobre a causalidade do adoecimento, tais como: a relação entre a espiritualidade e a saúde, a importância do trabalho multidisciplinar e da intersetorialidade versus o atual medicalocentrismo e as redes de atenção à saúde em contrapartida ao hospitalocentrismo.

Os resultados da pesquisa demonstraram que a formação médica propiciada pelas IES dos participantes lhes propiciou um contato conceitual com o modelo ampliado de saúde, o que pôde ser evidenciado pelo aumento de evocações e de conteúdo das respostas sobre a causalidade do adoecimento correlacionadas a aspectos sociais, psicológicos, familiares e espirituais. A evocação da palavra "biopsicossocial" pelos acadêmicos do $6^{\circ} \mathrm{e}$ $12^{\circ}$ períodos demonstra uma aproximação com o conceito, entretanto, observa-se que esta ainda é tênue, pois as reflexões detalhadas e aprofundadas sobre o modelo ampliado foram escassas nas respostas. Evidenciou-se, também, que as palavras relacionadas ao modelo ampliado de saúde não permaneceram na representação dos acadêmicos de forma duradora, oscilando de acordo com a proximidade da abordagem dos conteúdos relacionados à saúde ampliada trabalhados nas disciplinas ou unidades curriculares do curso.

Com base nesse estudo, aponta-se para a necessidade de que os sentidos de saúde e doença sejam abordados de forma aprofundada durante a formação médica, objetivando a compreensão por parte dos acadêmicos quanto à sua importância e relevância, bem como para a ampliação da capacidade reflexiva e crítica frente ao tema.

\section{Referências}

ABRIC, J. C. A. Abordagem estrutural das representações sociais. In: MOREIRA, A. S. P. M.; OLIVEIRA, D. C. (Orgs.). Estudos interdisciplinares de representação social. Goiânia: A. B., 2000. p.27-38.

BARDIN, L. Análise de Conteúdo. Tradução: Luís Augusto Pinheiro. São Paulo: Edições 70, 2016. 
BRAGHETTA, C. C. Desenvolvimento e validação de um instrumento para avaliar espiritualidade: Escala de Atitudes Relacionadas à Espiritualidade (ARES). 2017. Dissertação. São Paulo: Faculdade de Medicina, Universidade de São Paulo, 2017.

BRASIL. Ministério da Educação. Conselho Nacional de Educação. Câmara de Educação Superior. Resolução CNE/CES No 4, de 7 de novembro de 2001. Institui diretrizes curriculares nacionais do curso de graduação em medicina. Diário Oficial da União. Brasília, 10 Nov 2001; Seção 1, p. 38.

BRASIL. Ministério da Educação. Conselho Nacional de Educação. Câmara de Educação Superior. Resolução CNE/CES $n^{\circ} 3$, de 20 de junho de 2014. Institui diretrizes curriculares nacionais do curso de graduação em medicina. Diário Oficial da União. Brasília, 23 Jun. 2014. n. 117, Seção 1, p. 8-11.

BULGARELI, J. L. G.; GUERRA, L. M.; MIRANDA, L.; PENEDO, R. M.; FREDDO, S.; GONDINHO, B. V. C.; CORTELlAZZI, K. L.; PROBST, L. F.; POSSOBON, R. F.; PEREIRA, A. C. Compreensão dos sentidos atribuídos ao processo saúde e doença num grupo focal de adolescentes: um estudo qualitativo na perspectiva fenomenológica, CIAIQ, 2017.

CAMARGO, B. V.; JUSTO, A. M. Iramuteq - um software gratuito para análise de dados textuais. Temas em Psicologia, Ribeirão Preto, v.21, n.2, p. 513-528, 2013.

CROMACK, L. M. F.; BURSZTYN, I.; TURA, F. R. O olhar do adolescente sobre saúde: um estudo de representações sociais. Ciência \& Saúde Coletiva, Rio de Janeiro, v.14, n. 2, p. 627-634, 2006.

CZERESNIA, D.; MACIEL, E. M. G. S.; OVIEDO, R. A. M. Os sentidos da saúde e da doença. Rio de Janeiro: Editora Fiocruz, 2013.

FAYERS, P. M. D. Quality of life. Assessment, analysis and interpretation. Chichester: John Wiley, 2000.

FERREIRA, A. B. H. Novo dicionário Aurélio da língua portuguesa. 4. ed. Curitiba: Ed. Positivo, 2009.

FLAMENL, C. L'analyse de similitude: une technique pour les recherches sur les représentations sociales. ln: Doise, W.; Palmonari, A (Org.). L'atude des representations sociales. Neuchâtel: Delachaux et Niestlé, 1986. p. 139-156.

GROSSI, F. R. S. Saúde e Doença: Um estudo das representações sociais de famílias rurais em um município de Mato Grosso do Sul. Dissertação. (Mestrado em Psicologia). Escola de Psicologia da Universidade Católica Dom Bosco, Campo Grande, MS., 2012.

LUCCHETTI, G.; LUCCHETTI, A. L.; ESPINHA, D. C.; OLIVEIRA, L. R.; LEITE, J. R.; KOENIG, H. G. Spirituality and health in the curricula of medical schools in Brazil. BMC Med Educ. 2012. 
MOSCOVICI, S. Representações sociais: investigações em psicologia social. Tradução Pedrinho A. Guareschi.11 ed. Petrópolis. RJ: Vozes, 2015.

OLIVEIRA, D.C.; SÁ, C.P. Representações sociais da saúde e doença e implicações para o cuidar em enfermagem: uma análise estrutural. R. Bras. Enferm. Brasilia, v. 54, n.4, p. 608-622, 2001.

REGINATO, V.; BENEDETTO, M. A. C.; GALLIAN, D. M. C. Espiritualidade e saúde: uma experiência na graduação em medicina e enfermagem. Trab. Educ., vol.14, n.1, pp.237-255. ISSN 1678-1007, 2016.

ROTTA, M. C. B. Representações sociais de saúde, doença, velho e velhice para idosos atendidos em uma Unidade Básica de Saúde. Dissertação. Campo Grande, MS. 2009.

SHIMIZU, H. E.; SILVA, J. E.; MOURA, L. M.; BERMÚDEZ, X. P.; ODEH, M. M. A estrutura das representações sociais sobre saúde e doença entre membros de movimentos sociais. Ciênc. Saúde coletiva. Rio de Janeiro, v. 20, n. 9, p. 2899-2910, set. 2015.

WACHELKE, J.; WOLTER, R. Critérios de construção e relato da análise prototípica para representações sociais. Psicologia: teoria e pesquisa. Brasília. v.27, n. 4. p. 521-526, 2011.

WORLD HEALTH ORGANIZATION. A glossary of terms for community health care and services for older persons. WHO Centre for Health Development, Ageing and Health Technical Report, volume 5, 2004. 